\title{
Chronic Pruritus Revealing Angioimmunoblastic T-Cell Lymphoma
}

\author{
El Anzi 0*, Maouni S, Sqalli A, Meziane M, Ismaili N, Benzekri L, \\ Senouci K and Hassam B \\ Department of Dermatology-Venereology, Ibn Sina University Hospital, Mohammed \\ V University, Morocco
}

*Corresponding author: El Anzi Ouiam, Department of Dermatology-Venereology,

Ibn Sina University Hospital, Mohammed V University, Rabat, Morocco, Tel : 002126 2460 4105; Email: elanzi.ouiam@gmail.com

\section{Abstract}

Angioimmunoblastic T-cell lymphoma (AITL) is an uncommon systemic lymphoproliferative disorder that comprises $18 \%$ of all peripheral T cell lymphomas. We report a case of AITL discovered during a paraclinical assessment of chronic pruritus.

Keywords: Angioimmunoblastic T-cell lymphoma; Cutaneous; Pruritus

\section{Introduction}

Angioimmunoblastic T-cell lymphoma (AITL) is an uncommon systemic lymphoproliferative disorder that comprises $18 \%$ of all peripheral $\mathrm{T}$ cell lymphomas [1]. Cutaneous manifestations in AITL are dominated by the macular papular rash. Other manifestations, such as pruritus, are more rarely observed. We report a case of AITL discovered during a workup of chronic pruritus.

\section{Case Report}

A 70 years old male was admitted to the dermatology department for 2 years history of generalised chronic pruritus associated with constitutional symptoms (fever, weight loss, and night sweats). Dermatological examination revealed scraping lesions such as excoriated papules and linear striations as well as physical examination found generalized lymphadenopathy and ascites but no hepatosplenomegaly or pleural effusion. Biological examinations showed leukocytosis, anemia and elevated lactate dehydrogenase. Skin biopsy revealed a leukocytoclastic vasculitis. An excisional biopsy of an inguinal lymph node demonstrated lymphocytic infiltrate with histiocytes, eosinophils, neutrophils, and plasma cells. Follicular hyperplasia was not evident, but interfollicular regions were expanded with proliferating vessels. Atypical lymphocytes showed strong positivity for CD5, CD10, and CD30 at immunohistological staining. The patient was diagnosed with AITL and was treated with chemotherapy.

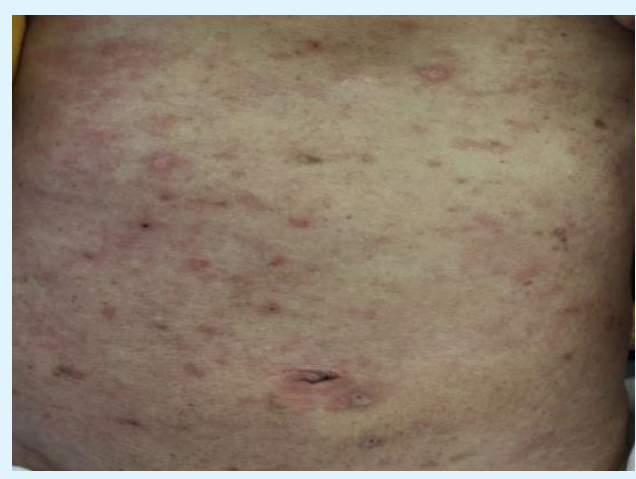

Figure 1: Scratch lesions. 


\section{Medical Journal of Clinical Trials \& Case Studies}

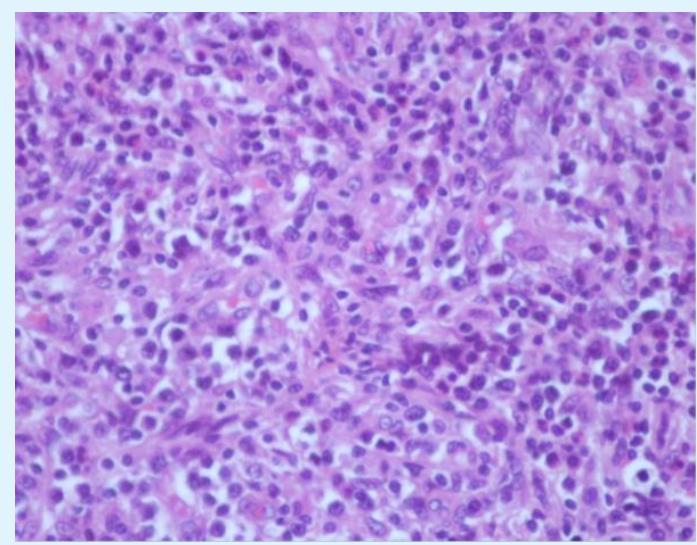

Figure 2: Biopsy of an inguinal lymph node.

\section{Discussion}

AITL is a rare systemic lymphoproliferative disorder but represents one of the most frequent subtypes $18 \%$ of peripheral $\mathrm{T}$ cell lymphomas [2]. Clinical aspect associates generalized lymphadenopathy, fever, weight loss, hepatosplenomegaly, pleural effusion, ascites and nonspecific cutaneous involvement such as morbilliform eruptions, urticarial, purpura and pruritus. Polyclonal hypergammaglobulinemia, elevation of Lactate Dehydrogenase and Anemia are the most commonly found biological abnormalities. Histopathology is fundamental for diagnosis. The most common finding is a superficial perivascular infiltrate with or without atypical lymphocytes expressing CD2, CD3, CD4, CD5, and occasionally CD8 [1,3]. A deficiency of cellular immunity explains the frequent occurrence of infections [3].

1st line treatment of $\mathrm{AI}$ T-cell lymphoma is Anthracycline-based therapy, including: (cyclophosphamide, doxorubicin, vincristine, and prednisone), but infectious complications are frequent $[1,3]$. Newer treatments have been applied including: rituximab (anti-CD20 therapy that targets B cells); cyclosporine, interferon, bevacizumab, thalidomide; romidepsin; and alemtuzumab (which targets CD52 on B and $\mathrm{T}$ cells). Autologous bone marrow transplantation is proposed to the youngest patients [1,3,4]. AI T-cell lymphomas have a very unfavorable prognosis with an overall survival of 26 to $36 \%$ at 5 years [5].

\section{Conclusion}

AITL must be considered if an elderly patient presents with a generalized pruritus or pruritic rash in the context of lymphadenopathy, fever, pleural effusions, and recent infections.

\section{References}

1. Min MS, Yao J, Kim SJ (2015) Development of a reticular rash in a febrile woman: An unusual cutaneous presentation of angioimmunoblastic T-cell lymphoma. JAAD Case Reports 1(3): 160-162.

2. Priyadarshini Pathak, Stuthi Perimbeti, Ashley Ames, Alison J Moskowitz (2019) Guillain Barré syndrome heralding the diagnosis of angioimmunoblastic T-cell lymphoma. Leukemia \& Lymphoma.

3. Florence Lachenal (2007) Lymphomes $\mathrm{T}$ angioimmunoblastiques Angioimmunoblastic T-cell lymphoma. La Presse Médicale 36(11): 1655-1660.

4. Joly L, Rosenstingl S, Huillard O, Gatfossé M (2008) Importante plasmocytose sanguine et médullaire réactionnelle à un lymphome angioimmunoblastique. La Revue de médecine interne 29(S1): 131.

5. Antoine C, Fongue J, Abed S, Okhremchuk I, Sahuc P, et al. (2015) Un DRESS atypique révélateur de lymphome $\mathrm{T}$ angio-immunoblastique. La Revue de médecine interne 36(1): A86. 